\title{
Genome-wide transcriptomic variations of human lymphoblastoid cell lines: insights from pairwise
} gene-expression correlations

\begin{abstract}
Aims: Human lymphoblastoid cell lines (LCLs) are a rich resource of information on human interindividual genomic, transcriptomic, proteomic and phenomic variations, and are therefore gaining popularity for pharmacogenomic studies. In the present study we demonstrate that genome-wide transcriptomic data from a small LCL panel from unrelated individuals is sufficient for detecting pairs of genes that exhibit highly correlated expression levels and may thus convey insights about coregulated genes. Materials \& methods: RNA samples were prepared from LCLs representing 12 unrelated healthy adult female Caucasian donors. Transcript levels were determined with the Affymetrix Human Gene arrays. Expression-level correlations were searched using Partek ${ }^{\circledR}$ Genomics Suite ${ }^{\mathrm{TM}}$ and the R environment. Sequences of detected correlated gene pairs were compared for shared conserved 3'-UTR miRNA binding. Results: Most of the approximately 33,000 transcripts covered by the Affymetrix arrays showed closely similar expression levels in LCLs from unrelated donors. However, the expression levels of some transcripts showed large inter-individual variations. When comparing the expression levels of each of the top 1000 genes showing the largest interindividual expression variations against the others, two sets containing 156 and 4438 correlated gene pairs with false-discovery rates of 0.01 and 0.05 were detected, respectively. Similar analysis of another gene-expression data set from LCLs (GSE11582) indicated that 61 and 39\% of identified pairs matched the pairs detected from our transcriptomic data, respectively. Shared conserved 3'-UTR miRNA binding sites were noted for $14-17 \%$ of the top 100 gene pairs, suggesting that regulation by miRNA may contribute to their coordinated expression. Conclusion: Probing genome-wide transcriptomic data sets of LCLs from unrelated individuals may detect coregulated genes, adding insights on cellular regulation by miRNAs.
\end{abstract}

Original submitted 11 July 2012; Revision submitted 4 September 2012

\section{KEYWORDS: Affymetrix gene-expression arrays bioinformatics Epstein-Barr virus GUSB human lymphoblastoid cell lines LCLs miRNA RPL5 RPL10 RPL18 transcriptomics}

Human lymphoblastoid cell lines (LCLs) immortalized by the Epstein-Barr virus (EBV) are gaining increased popularity as a research tool for the study of human genomic, epigenomic and phenomic variations and are therefore often utilized for pharmacogenomic studies [1-5]. EBV-immortalized LCLs are utilized by the HapMap Project [3] and other international collaborative projects on human genome variation, and many biobanks include large LCL repositories from healthy or diseased individuals as a primary biomedical research tool [1-5].

LCLs are generated from peripheral blood B lymphocytes and retain most of their phenotypic properties, including the production of antibodies. Moreover, the EBV genome does not incorporate with nuclear DNA meaning that the nuclear genomic sequence remains intact, faithfully representing the donor's sequence. The transcriptomes of
LCLs encompass a large portion of human genes from diverse cellular pathways [6] and have been valuable for studying genome-wide individual differences in transcriptomes [7] and in alternative mRNA splicing [8]. Thus, human LCL transcriptomes faithfully reflect heterogeneity specific to the individuals from whom the cells have originated. LCLs have also proved valuable for studies on pharmacogenomic gene-expression markers, for example, for the cancer drugs cisplatin, etoposide, $N$-methyl- $N$-nitro- $N$-nitrosoguanidine, gemcitabine and cytosine arabinoside [9-14], as well as for sensitivity to radiation [15]. Of note, Choy and coworkers have identified batch effect and cellular growth rates as major confounders for some of these findings [16]. Therefore, transcriptomic studies on drug effects in LCLs should be viewed as preliminary until their validation with clinical samples.

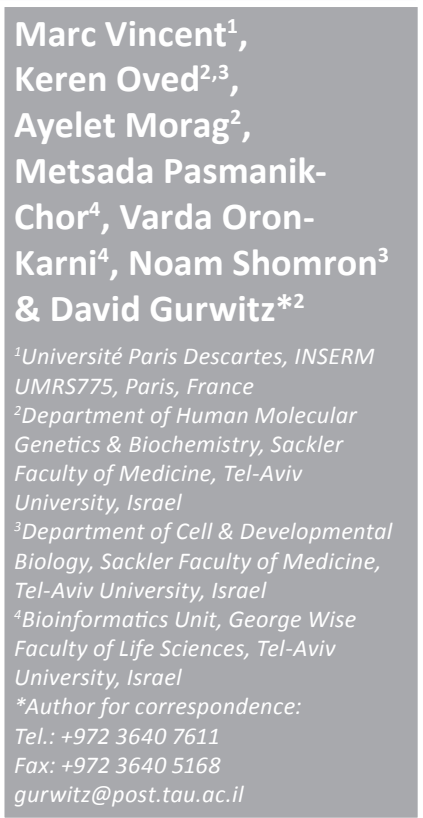

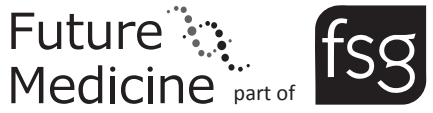


Recently, we have employed LCLs from unrelated healthy individuals as tools for discerning shared drug pathways [17], searching for drugresponse biomarkers for antidepressant serotonin-selective reuptake-inhibitor drugs [18,19] and for studying sex differences in adverse drug events [20]. LCLs have also been constructive for discovering genes and metabolic pathways implicated in severe diseases such as autism [21].

Studies of gene-expression correlations have proven to be a robust tool for reconstructing gene network pathways [22-24]. Eisen and coworkers have introduced the cluster analysis of genome-wide expression patterns and demonstrated that the method can be applied for gaining insights about the function of poorly characterized genes according to their expression correlations with well-known genes [22]. Numerous studies have subsequently used similar clustering approaches for studying gene ontology and gene-expression changes in various pathological states. In the present article, we describe the potential of genome-wide transcriptomic data sets from LCLs representing unrelated healthy donors for exploring pairwise correlations of gene-expression levels.

\section{Materials \& methods Cells}

LCLs from 12 consenting healthy adult female donors of Caucasian ancestry were obtained from the National Laboratory for the Genetics of Israeli Populations at Tel-Aviv University (Israel) [101] as previously described [17-20]. The cell lines were immortalized from lymphocytes of healthy adult donors using the same stocks of B-95-derived EBV and with the same immortalization protocol. Cells were maintained in RPMI medium, supplemented with $10 \%$ fetal bovine serum and antibiotics $(100 \mathrm{U} / \mathrm{ml}$ penicillin; $100 \mu \mathrm{g} / \mathrm{ml}$ streptomycin), and kept at a temperature of $37^{\circ} \mathrm{C}$, with $6 \% \mathrm{CO}_{2}$ and $100 \%$ humidity. All cell culture reagents were purchased from Biological Industries (Israel).

\section{RNA extraction}

RNA extraction was performed from cells incubated in T-25 flasks under optimal growth conditions (exponential growth). Cells were centrifuged and then lyzed using Tri-reagent (T9424 ${ }^{\circledR}$, Sigma-Aldrich, MO, USA), followed by RNA separation using chloroform and precipitation using isopropanol, total RNA purification was achieved using phenol-chloroform extraction and Phase Lock Gel ${ }^{\mathrm{TM}}$ tubes (light $2 \mathrm{ml}$; Fisher Scientific, NH, USA) [25]. RNA quality was checked using RNAse-free, 1\% agarose gel, and was quantified using a nanodrop spectrophotometer (ND-1000; Fisher Scientific). The spectrophotometric absorbance parameters of the samples were: $260 / 280 \mathrm{~nm}>2.0$ and $260 / 230 \mathrm{~nm}>2.0$.

\section{Microarray experiment \& microarray data analysis}

Twelve Affymetrix (CA, USA) GeneChip ${ }^{\circledR}$ Human Gene 1.0 ST arrays were used for geneexpression analysis according to the instruction manual, as described in the Affymetrix website [102]. Human Genome Organisation (HUGO)compliant probe set annotation was retrieved from the Affymetrix website (latest release 32 ). Microarray analysis was performed on CEL files using Partek ${ }^{\circledR}$ Genomics Suite ${ }^{\mathrm{TM}}$ (Partek Inc., MO, USA) [103]. Batch effect removal was applied for the different samples, to remove individual variations. All expression units are displayed as raw expression data (Affymetrix arbitrary units in $\log _{2}$ scale). Gene-gene correlations and corresponding plots were obtained with the $\mathrm{R}$ environment using linear least-square fitting. First, we selected the 1000 probe sets that showed the highest gene-expression-level variation. We then considered the set of 499,500 pairs that could be generated from these 1000 probe sets. From this set, we kept only the pairs where each of the probe sets annotated with coding genes unambiguously attributed to the same gene family (i.e., annotation of each of the probe sets included one gene family only; this step was essential for removing probe sets whose annotations were ambiguous). Since our goal was to find novel unreported gene pairs, we proceeded to crude filtering of probe set pairs that we considered obvious or uninformative, as both pair members belong to the same gene family. Our criterion to decide a-priori if a probe set pair was uninformative was to check whether the probe sets targeted the same genes or genes belonging to the same family. Gene families were retrieved from HUGO gene annotations using mapping files available for download at the HUGO gene nomenclature website [104]. We added supplementary mapping rules to regroup immunoglobulin genes in a corresponding superfamily.

At the end of the filtering procedures we obtained 410,557 probe set pairs corresponding to 275,189 gene pairs produced from 887 genes. We proceeded to analysis with these remaining pairs, and their expression signals in the 12 LCLs were compared pairwise. The correlation of two probe sets was assessed using a two-sided 
statistical test based on the Pearson correlation coefficient, as available in the 'cor.test' routine from the 'stats' package in the $\mathrm{R}$ environment. The statistical significance of the correlations was characterized using the t-statistic and computing the corresponding $\mathrm{p}$-value. In order to select sets of pairs to be further studied, we chose a level of significance $\alpha$ to be used with p-values that were corrected for multiple testing using appropriate procedures. Namely, to obtain two large sets with small expected false discovery rate (FDR) we used the Benjamini and Hochberg step-up FDR control procedure [26]. To obtain a small list of gene pairs with low probability of containing false positives we used a Bonferroni control procedure. We denote by $\alpha$ the level at which we control either the FDR or the family-wise error rate.

\section{Common miRNA binding sites for paired genes}

Sequences of paired genes showing transcriptome correlations were analyzed for the presence of shared evolutionally-conserved 3'-UTR miRNA binding sites. For each gene pair we first predicted miRNAs that target each gene using DIANA microT v3.0 software $[27,28,105]$ and TargetScanHuman 5.2 [106]. The DIANA-microT 3.0 and TargetScanHuman 5.2 algorithms are based on parameters that are calculated individually for each miRNA, and for each miRNA recognition element, depending on binding and conservation levels. The total predicted score is calculated as the weighted sum of conserved and nonconserved miRNA recognition elements on the 3'-UTR of a gene [27,28]. For DIANA microT v3.0, a higher score corresponds to higher possibility of targeting the gene; in our predictions we used score threshold of 7.3, which is the software default [105]. We subsequently compared between the miRNAs predicted to target one gene of each pair with the miRNAs predicted to target the other pair member, and searched for miRNAs targeting both pair members. Lastly, data were filtered by analyzing shared miRNA binding sites with either software $[105,106]$.

\section{Comparison with web resources of human transcriptomics data}

Data from our microarray experiments were compared with data from the Gene Expression Omnibus on the NCBI server [107]. Topexpressed genes and those showing the highest expression level standard deviations among individual LCLs were searched using the Geo Data Set browser interface [107]. The search was limited to expression data derived from LCLs from healthy donors and using the Affymetrix or Illumina (CA, USA) arrays. One data set (GSE11582; [108]) from the HapMap Project corresponding to cell lines from 269 unrelated CEPH individuals was found to be suitable for comparison. From this data set, we used the 83 LCLs corresponding to donors of European ancestry. This data set is based on Affymetrix Human Genome U133A arrays. The comparison was performed using the CEL files available from the website, and analysis by Partek Genomics Suite [103] as previously described [18,19]. In order to proceed with the comparison, we selected the gene pairs associated with probe sets pairs showing a correlation with a Benjamini and Hochberg (BH)-FDR-corrected p-value either under level $\alpha=0.05$ (BH-FDR large set) or $\alpha=0.01$ (BH-FDR small set) when using data from the 12 LCLs. We then found the corresponding genes and probe sets pairs in the GSE11582 data set using Affymetrix's latest annotation files for the U133A arrays and performed a correlation analysis using the GSE11582 expression data. For each original gene pair we then obtained a raw $\mathrm{p}-\mathrm{Val}_{\min }$ measure equal to the minimum $\mathrm{p}$-value observed over all corresponding probe set pairs. Equivalently, we obtained $\mathrm{r}_{\text {max }}^{2}$ measures for the Pearson correlation coefficients. These aggregate measures were necessary owing to the large numbers of relationships between gene pairs and probe set pairs after filtering. In order to assess the significance of these aggregated $\mathrm{p}$-values and the Pearson correlation coefficients, we sampled pairs of unrelated genes from the GSE11582 data set and measured their respective $\mathrm{p}-\mathrm{Val}_{\min }$ and $\mathrm{r}_{\text {max }}^{2}$. We repeated the sampling ten times for $n \alpha$ gene pairs, where n $\alpha$ is the number of gene pairs we had previously retrieved in the GSE11582 data set at given level.

\section{Novelty check using interaction databases}

The 4438 gene pairs that were found using the gene-expression data sets from our 12 LCLs following the filtration steps were programmatically searched in two protein interactions databases, iRefIndex [29,109] and STRING $[30,31,110]$. For the iRefIndex search, the HUGO gene annotations were converted to uniprot annotations using mapping files available form the HUGO website [104]. Of 666 initial genes that are present in the large list of gene pairs (Supplementary Table 1; see www.futuremedicine. com/doi/suppl/10.2217/pgs.12.179), 628 were found to have corresponding uniprot identifiers 
and could be examined in these protein interactions databases. The iRefIndex database was then downloaded and queried using the iRefR package from the $R$ environment. To recover interactions from the STRING database we used the STRING web API [110]. As a first step, we queried the API to obtain STRING identifiers from the HUGO annotations. Next, 640 of the 666 initial genes were found to have corresponding entries in STRING. A second query, again employing the STRING database, was carried out using the obtained identifiers in order to retrieve interactions of interest. For each database, the set of interactions was constrained to represent human proteins.

\section{Results \& discussion \\ Gene pairs \& their tentative coregulation by miRNAs}

When analyzing the basal transcriptomes of individual LCLs representing 12 unrelated female donors, most of the approximately 33,000 transcripts covered by the Affymetrix arrays showed closely similar expression levels. However, 125 transcripts coding for known genes showed variations of more than twofold ( $>1$ Affymetrix raw expression units in $\log _{2}$ scale), as determined with the Affymetrix Human Gene 1.0 ST arrays. TABLE 1 presents the mean expression levels and standard deviations for the top 25 genes with the highest interindividual expression levels variations. As shown, for some genes the expression levels vary by over 50 -fold between LCLs with high- and low-expression levels. Notably, five of the ten top genes in this list (IGHD, IGKV3D-11, IGKC, VSIG6 and IGHM) code for immunoglobulin family proteins. The correlations in their individual expression levels are not surprising as they reflect inherent recombination during B-cell maturation; nonetheless, these correlations demonstrate the power of our computational approach. For a full list of transcript level variations within the same LCLs panel see Supplementary Table 2.

The 15 most notable correlations found between paired genes are shown in Table 2 \& Figure 1 . This list was constructed by comparing the expression levels of each of the 1000 most variable transcripts in each of the 12 unrelated individuals with each other, followed by filtration as described under 'Materials \& methods'. It is evident that some expression level correlations for paired genes are very high, as also illustrated by the examples presented in Figure 2 . All 15 pairs presented have a Bonferroni corrected $\mathrm{p}<0.05$.

The examples shown in Figure 2 demonstrate that high expression level correlations were also observed for genes located on different chromosomes - apparently reflecting coregulation by the same transcription factor or miRNAs. A search for possible shared miRNAs which may bind to conserved 3'-UTR regions of both genes in each of the top 100 gene pairs was performed using the DIANA-microT 3.0 algorithm $[27,28,105]$ and TargetScanHuman 5.2 [106] software (see 'Materials \& methods' section). These searches yielded 17 and 14 pairs, respectively, where the same miRNAs were predicted to bind and apparently regulate the expression of both pair members, as presented in Table 3. That is, our search has detected common miRNAs binding sites for 17 and $14 \%$ of the top 100 gene pairs, using DIANA and TargetScan software, respectively. The two software tools agreed for 11 of these 100 top gene pairs (Table 3). There is a continuous debate in the miRNA field about the reliability and the methods used for target-gene predictors. While this is beyond the scope of this study, we found it appropriate to investigate the shared miRNA binding sites for the correlated expressed pairs of genes. We did not want to rely on merely one target predictor as this might bias us to one method for target selection. Therefore, we chose two of the most popular ones (out of many others available). The sometimes low overlap in the targets between the two methods indicates the different features each method uses to detect target genes. For example, one tool might rely on conserved targets while the other on nonconserved ones. Thus, combining the output of the two sets of target miRNA allows the reader to gain insight into two approaches to identify these sets and does not necessarily weaken any of the conclusions regarding these overlaps. If one wants to be on the cautious side then one should take only the overlapping miRNA for further studies, and for this purpose these are also shown in Table 3 .

Of note, over half of human genes are estimated to be regulated by miRNAs, and each miRNA is assumed to regulate the expression of several genes [32,33]. Interestingly, in the context of pharmacogenomics, miRNAs were suggested to be more commonly implicated in the regulation of genes relevant for drug pharmacodymamics as compared with drug pharmacokinetics [34].

The list of gene pairs with highly correlated transcript levels yielded some surprising gene pairs not known to share a biological pathway. For example, a striking correlation with $\mathrm{p}=4 \times 10^{-9}$ and Pearson correlation coefficient $r^{2}=0.98$ with was noted for the expression of the orphan G-protein-coupled receptor GPRC5D 
(located on chromosome 12p13.3) and AKAPG (PRKA; located on chromosome 14q12). Likewise, a correlation with $\mathrm{p}=7 \times 10^{-8}$ and Pearson correlation coefficient $\mathrm{r}^{2}=0.97$ was observed for the expression of CHL1 (located on chromosome $3 \mathrm{p} 26.1$ ) and CRIM1 (located on chromosome 2p21); while the proteins coded by the latter two genes are both implicated in tissue development $[35,36]$, they are not known to share a cellular pathway.

Further examples of gene pairs exhibiting high expression level correlations (small and large BH-FDR sets with FDR controlled at level $\alpha=0.01 \alpha=0.05$ ) in LCLs from 12 unrelated individuals are presented in Supplementary Table 1 \& Supplementary Tabee 3 containing 156 and 4438 gene pairs, respectively. It is noteworthy that the expression levels are positively correlated for the majority of such gene pairs: from the top 100 correlations (by $\mathrm{r}^{2}$ value) only six gene pairs $(6 \%)$ show negative expression level correlations. This observation suggests that these top correlations are unlikely to represent chance findings, in which case one would expect to observe equal numbers for positive and negative expression level correlations for gene pairs. This conclusion is further supported by the finding that when examining the large BH-FDR set of 4438 gene pairs, the proportion of negative correlations rises from only 6 to $25 \%$ of all gene pairs. Moreover, the high representation of positive correlations suggests that the transcription of most detected gene pairs is coregulated by shared transcription factors and/or miRNAs.

\section{Novelty of the detected gene pairs}

With the large BH-FDR list of 4438 gene pairs we proceeded to query protein interaction databases to examine whether these pairs were novel when compared with protein interactions included in the interaction databases iRefIndex and STRING (see 'Materials \& methods'). In the iRefIndex database, no known interactions were found for any of these 4438 gene pairs, while only $29(0.63 \%)$ gene pairs had corresponding interactions in the STRING database. These results are better interpreted keeping in mind that the two protein-centric databases include additional interaction types besides direct physical protein-protein interactions. More specifically, they both include known genetic interactions and the STRING database also includes functional associations inferred from scientific literature text-mining (both cocitations and natural language processing), as well as coexpression analysis [30,31]. From this
Table 1. Genes exhibiting high expression level variations among human lymphoblastoid cell lines from unrelated individuals.

\begin{tabular}{|c|c|c|c|}
\hline Gene & Average $^{\dagger}$ & SD & Range (fold difference) \\
\hline IGHD & 10.60 & 2.53 & 5.17-12.41 (104) \\
\hline UGT2B $7^{\ddagger}$ & 7.58 & 2.40 & 2.69-10.12 (172.4) \\
\hline IGKV3D-11 & 6.25 & 2.11 & $3.02-10.3(155.4)$ \\
\hline PLOD2 & 6.14 & 1.95 & $3.41-8.85(43.4)$ \\
\hline IGKC & 6.5 & 1.92 & $3.28-9.43(71)$ \\
\hline NRN1 & 6.94 & 1.88 & $4.29-10.0(52.3)$ \\
\hline VSIG6 & 6.84 & 1.86 & $4.95-10.72(54.6)$ \\
\hline IGHM & 9.67 & 1.86 & $5.74-11.59$ (57.7) \\
\hline$H L A-D Q A 1$ & 9.55 & 1.85 & $6.2-11.38(36.2)$ \\
\hline RGS13 & 7.09 & 1.84 & $3.69-9.46(54.6)$ \\
\hline UGT2B15 & 6.60 & 1.82 & $3.24-8.60(41.1)$ \\
\hline IGLJ3 & 10.43 & 1.82 & $7.14-12.36(37.3)$ \\
\hline IGHA1 & 10.56 & 1.79 & $7.32-12.53(37)$ \\
\hline NLRP2 & 4.92 & 1.75 & $2.82-7.33(22.8)$ \\
\hline LEPREL1 & 5.5 & 1.69 & $4.01-8.7(25.8)$ \\
\hline IGK & 6.36 & 1.65 & $3.98-9.35(41.3)$ \\
\hline$A P P$ & 6.66 & 1.64 & $3.53-8.84(39.7)$ \\
\hline FAM169A & 5.60 & 1.63 & $3.71-7.98$ (19.3) \\
\hline MMP7 & 5.02 & 1.60 & $3.01-7.61(24.3)$ \\
\hline CHI3L2 & 8.95 & 1.59 & $6.21-10.87(25.3)$ \\
\hline TC2N & 5.33 & 1.56 & $3.0-7.86(29)$ \\
\hline PLD1 & 7.31 & 1.55 & $4.11-9.12(32.2)$ \\
\hline KIAA1324L & 4.86 & 1.51 & $3.0-6.69(12.9)$ \\
\hline CHL1 & 6.99 & 1.50 & $4.32-8.76(21.7)$ \\
\hline GUCY1B3 & 7.86 & 1.49 & $4.3-9.57(38.6)$ \\
\hline \multicolumn{4}{|c|}{$\begin{array}{l}\text { Mean and SD expression values are presented for the top } 25 \text { genes whose expression varied the most } \\
\text { (highest SD) among the } 12 \text { individual human lymphoblastoid cell lines from unrelated female donors. } \\
\text { 'Expression values are shown in arbitrary Affymetrix units in } \log _{2} \text { scale (see text). See SUPPLEMENTARY } \\
\text { TABLE } 2 \text { for the entire data set. } \\
\text { "This entry represents transcript \#8100758 on the Affymetrix Human Gene } 1.0 \text { ST microarrays, which, } \\
\text { according to the Affymetrix website, detects UGT2B7. However, according to our analysis this } \\
\text { annotation seems to be incomplete, as the probes used for this transcript also perfectly match the } \\
\text { sequences of UGT2B10, UGT2B11, UGT2B15 and UGT2B17 (all are closely similar human genes). The } \\
\text { Affymetrix support team has been notified about this mistake. } \\
\text { SD: Standard deviation. }\end{array}$} \\
\hline
\end{tabular}

we infer that most of the gene pairs detected in our study represent novel associations compared with known interactions involving human genes and their protein products.

\section{Confirmation with an independent} human LCLs gene-expression data set

Considering the small number of transcriptomic data sets in the present study (12 arrays), and in order to confirm the significance of the detected gene pairs, we quantified to what extent their expression levels were correlated in an independent and by far larger LCLs genome-wide 
Table 2. Gene pairs exhibiting the highest correlations of expression levels in human lymphoblastoid cell lines from unrelated donors.

\begin{tabular}{|c|c|c|c|}
\hline First gene & Second gene & $\mathbf{r}^{2}$ & $\begin{array}{l}\text { Raw } p\left(p V a l_{\text {min }} \text { in }\right. \\
\left.\text { GSE11582 }^{+}\right)\end{array}$ \\
\hline$A K A P 6$ & GPRC5D & 0.9724 & $3.92 \times 10^{-9}\left(5 \times 10^{-3}\right)$ \\
\hline BEND4 & IFI44L & 0.9665 & $1.05 \times 10^{-8}$ \\
\hline HNF1B & EPS8 & 0.9661 & $1.12 \times 10^{-8}(\sim 0)$ \\
\hline CPNE4 & CYP27A1 & 0.9629 & $1.76 \times 10^{-8}$ \\
\hline SGPP2 & IFI44 & 0.9581 & $3.21 \times 10^{-8}$ \\
\hline CREB5 & NCKAP5 & 0.9542 & $5.04 \times 10^{-8}$ \\
\hline SLC39A10 & MYO1F & 0.9541 & $5.09 \times 10^{-8}$ \\
\hline CREB5 & $|F| H 1$ & 0.9538 & $5.27 \times 10^{-8}\left(6 \times 10^{-9}\right)$ \\
\hline BEND4 & SGPP2 & 0.9528 & $5.82 \times 10^{-8}$ \\
\hline LRRK2 & LMO3 & 0.9523 & $6.19 \times 10^{-8}$ \\
\hline CHL1 & CRIM1 & 0.9515 & $6.71 \times 10^{-8}\left(4 \times 10^{-15}\right)$ \\
\hline$|F| 44 L$ & SGPP2 & 0.9506 & $7.35 \times 10^{-8}$ \\
\hline SAMD9L & OAS2 & 0.9492 & $8.50 \times 10^{-8}$ \\
\hline OGFRL1 & $X Y L T 1$ & 0.9492 & $8.50 \times 10^{-8}\left(6 \times 10^{-5}\right)$ \\
\hline CYP27A1 & $L I P N$ & 0.9484 & $9.12 \times 10^{-8}$ \\
\hline \multicolumn{4}{|c|}{ 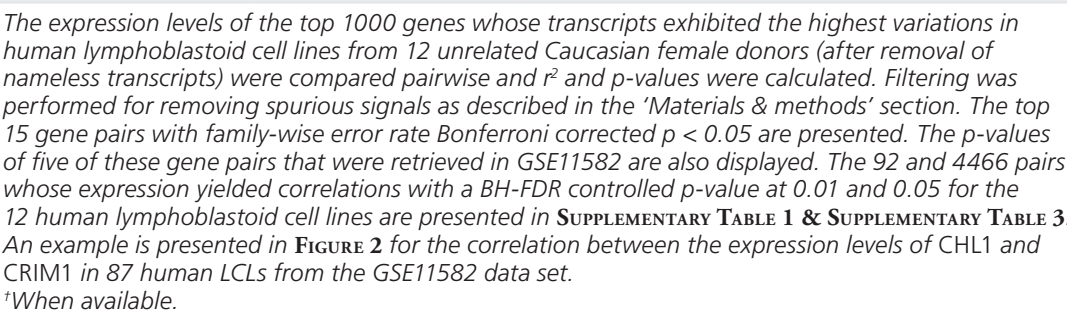 } \\
\hline
\end{tabular}

expression data set (GSE11582, see 'Materials and methods' section). When querying the GSE11582 human LCLs data set, and comparing with our two BH-FDR sets (FDR controlled at level $\alpha=0.01$ and $\alpha=0.05$, respectively), we retrieved 85 of our 156 pairs (54\%) and 2307 of our 4438 pairs (52\%; Suprlementary TABLE 4), respectively. These numbers are consistent with the fact that the GSE11582 data set was obtained using an earlier Affymetrix chip that covers approximately half of the transcriptome (Affymetrix GeneChip ${ }^{\circledR}$ Human Genome U133A), while our study used a later chip aimed at covering the whole transcriptome (Affymetrix GeneChip ${ }^{\circledR}$ Human Gene 1.0 ST). In these two BH-FDR sets 61 and 39\% of the pairs, respectively, had $\mathrm{pVal}_{\min }<0.05$ (adjusted for multiple testing using the Bonferroni procedure). The median $\mathrm{pVal}_{\min }$ of these retrieved pairs were $7.30 \times 10^{-6}$ and $2.33 \times 10^{-4}$ while their median $\mathrm{r}^{2}{ }_{\max }$ values were 0.47 and 0.39 , respectively. This comparison clearly showed a correlation enrichment when compared with ten random samples of $n_{\alpha}$ pairs from the same data set (with $\mathrm{n}_{0.05}=85$ and $\mathrm{n}_{0.01}=2307$ ), showing for $\alpha=0.05$ a median $\mathrm{pVal}_{\text {min }}$ of $0.18 \pm 0.02$ (intersample standard deviation) and a median $\mathrm{r}_{\text {max }}^{2}$ of $0.2 \pm 0.005$. For $\alpha=0.01$, as expected, the samples showed roughly similar median $\mathrm{pVal}-$ ${ }_{\min }$ of $0.14 \pm 0.04$ and $r^{2}{ }_{\max }$ of $0.22 \pm 0.01$. The same enrichment was observed when examining the proportion of pairs showing a $\mathrm{pVal}_{\text {min }}$ under the corrected significance level 0.05 in the ten random samples, which was only $1 \pm 0.5 \%$ $(\alpha=0.05)$ and $4 \pm 0.02 \%(\alpha=0.01)$. Thus, our examination of an unrelated human LCL genome-wide expression data set indicated that approximately half the gene pairs detected in our data using this approach are most likely true examples of human gene pairs with highly correlated expression levels. Gene pairs not confirmed in the independent larger data set may represent false hits, or pairs missed in the larger data set (GSE11582) because it represents CEPH cell lines prepared by several laboratories, unlike our data set, thus introducing batch effect [16].

\section{Future perspective}

The data presented here should be considered as preliminary. Definitely, more robust data on gene pairs with correlated transcript levels, and possibly more structured gene networks, may be derived from larger panels of LCLs or other tissues collected from larger numbers of unrelated individuals. In addition, analyzing genome-wide expression data for larger gene sets (e.g., triads) is desirable, but beyond the scope of the present manuscript. For the time being, the following points should be considered in favor or using the data as a preliminary reference for studies on gene-expression variations among LCLs from unrelated individuals. First, the 12 human LCLs used for preparing the RNA samples and estimating the genome-wide expression profiles all represent female donors of Caucasian ancestry; the cell lines were all immortalized by the same laboratory [101] and using the same stock of EBV; these are key considerations when studying drug-response phenotypes and related transcriptomes in human LCLs, as demonstrated by Choy and coworkers [16]. Inclusion of transcriptomics data from female donors only may be crucial, as variations were noted between the male and female human transcriptomes [37]. However, as shown above, appoximately half the gene pairs detected in our small female LCL cohort were also detected in the GSE11582 data set, which includes both male and female donors. In future, it would 
be useful to compare the current data with genome-wide expression profiles of LCLs from larger numbers of male and female donors. Second, our LCLs were all grown in parallel using similar growth conditions and the RNA samples were prepared and assayed in parallel with similar protocols. Such similar growth and assay conditions assure high reproducibility of the transcriptomics data and enhance its quality for comparisons between LCLs from individual donors.

Two additional considerations support the validity of the recorded observations. First, for most genes the standard deviations for measurements of expression levels in the RNA samples representing the 12 unrelated female donors are small $(\mathrm{SD}>1$ were observed only for 139 of 33,297 transcripts). Second, in a recent study, the raw expression data from the same microarrays were employed for finding 12 tentative gene-expression biomarkers for LCL sensitivity to growth inhibition by the antidepressant drug paroxetine, and in that study the Affymetrix array data for these 12 genes were validated by real-time PCR and found to be in close correlation with the raw microarray expression data [18].

The concept of cluster analysis using genome-wide expression profiling from microarray experiments performed in different cell lines, or in a given cell line studied in different physiological conditions, is well established [22-24]. However, we would like to point out that the pairwise expression correlation approach as applied in the present report allows certain advantages compared with cluster analysis. First, while the primary input of pairwise analysis and cluster analysis are both pairwise relations (correlation/distance) between geneexpression probes, in cluster analysis these relations are aggregated to yield clusters. This implies that when attempting validation we either have to consider each cluster as a whole or to consider the separate pairwise relations that constitute them. In the former case it may not always be possible to find the exact same cluster composition using the validation data set, while the latter case reduces the validation to pairwise analysis. Moreover, the choice of the aggregation technique used (e.g., singlelink hierarchical clustering) may add a bias on the choice of the clusters found and therefore complicate the interpretation of the results. Therefore, examining single pairs of geneexpression levels instead of clusters simplifies the definition, interpretation and validation of the relationships found between genes. However, hierarchical clustering of gene-expression data sets definitely has other advantages over pairwise analysis, as it allows the construction of elaborate interactome maps and may help uncover their underlying structure [22-24].

Data on the whole-genome transcriptomes of human LCLs representing unrelated donors are openly available in several web databases, such as Gene Expression Omnibus on the NCBI server [107]. However, to our knowledge, such data have not been discussed in the scientific literature for the purpose of showing pairwise gene-expression patterns of human genes, as presented in this article. We hope that this concept will be further applied for reconstructing gene networks, using LCL transcriptomic data sets. It would also be of interest to examine if unique gene pairs are detected when performing such analysis for female versus male donors, young versus older donors, or donors of different ethnic backgrounds.

Our data may in addition be of use for selecting sets of genes with high expression levels and minimal variations in LCLs representing unrelated donors. Examples for ten such genes are shown in Table 4. Of note, three of them

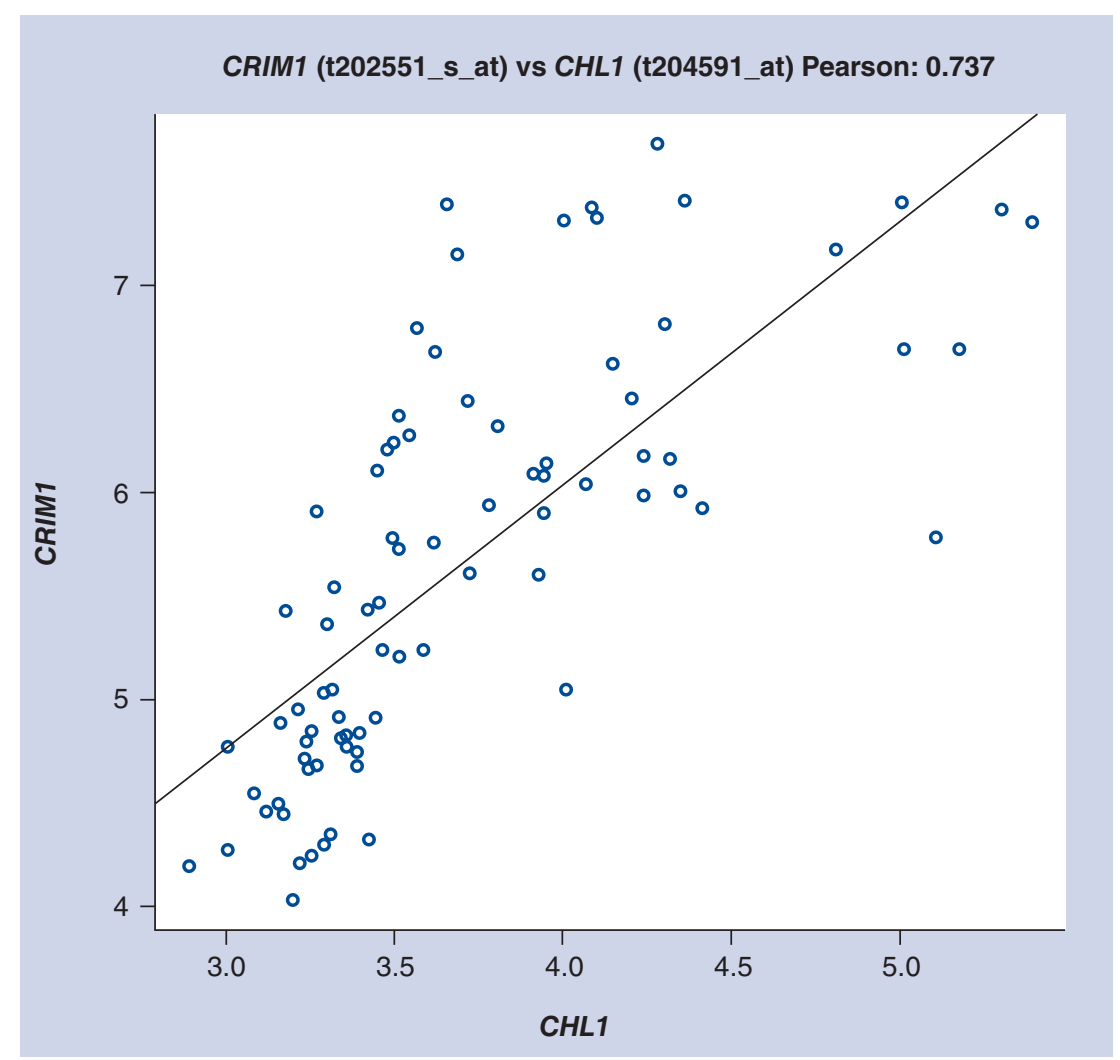

Figure 1. Pearson correlation plot between the expression levels of CHL1 and CRIM1 as determined in human lymphoblastoid cell lines from 87 unrelated individuals from the GSE11582 data set. 


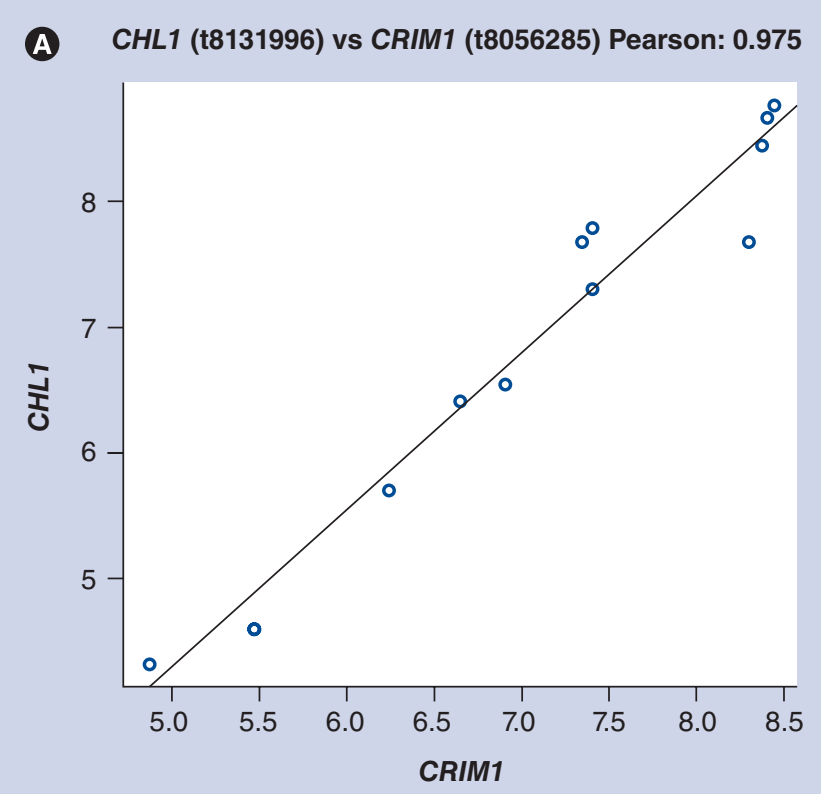

C SLC39A10 (t8077270) vs MY01F (t8041447) Pearson: -0.977

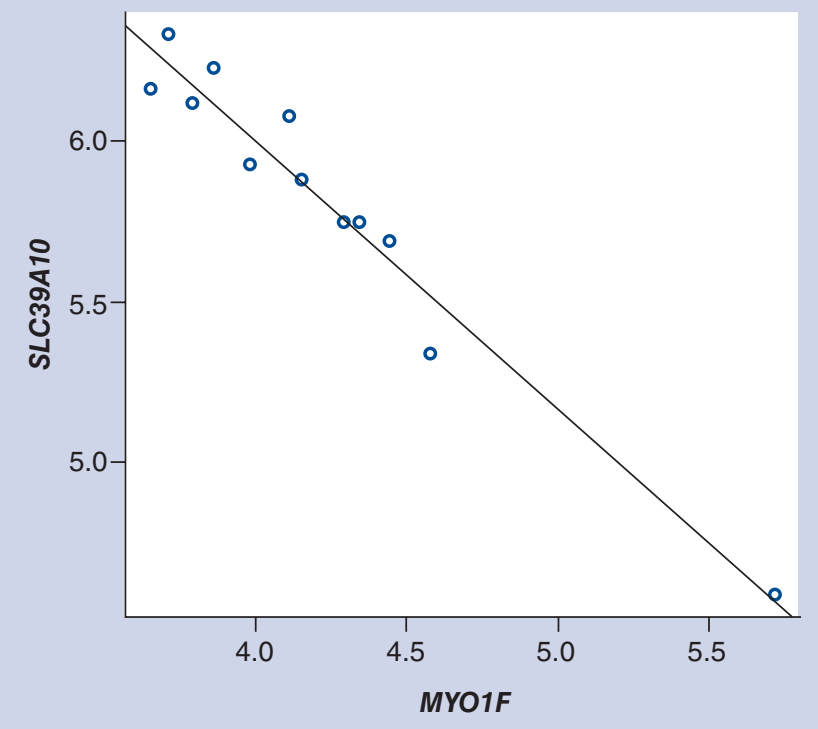

\section{B CREB5 (t8090690) vs IFIH1 (t8048432) Pearson: -0.977}

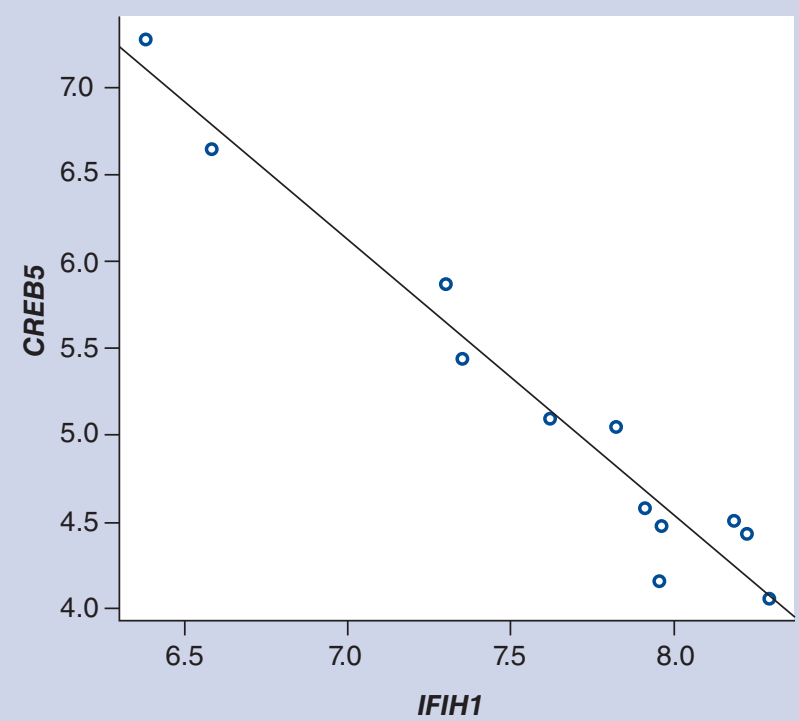

D CPNE4 (t8047174) vs CYP27A1 (t8033605) Pearson: 0.981

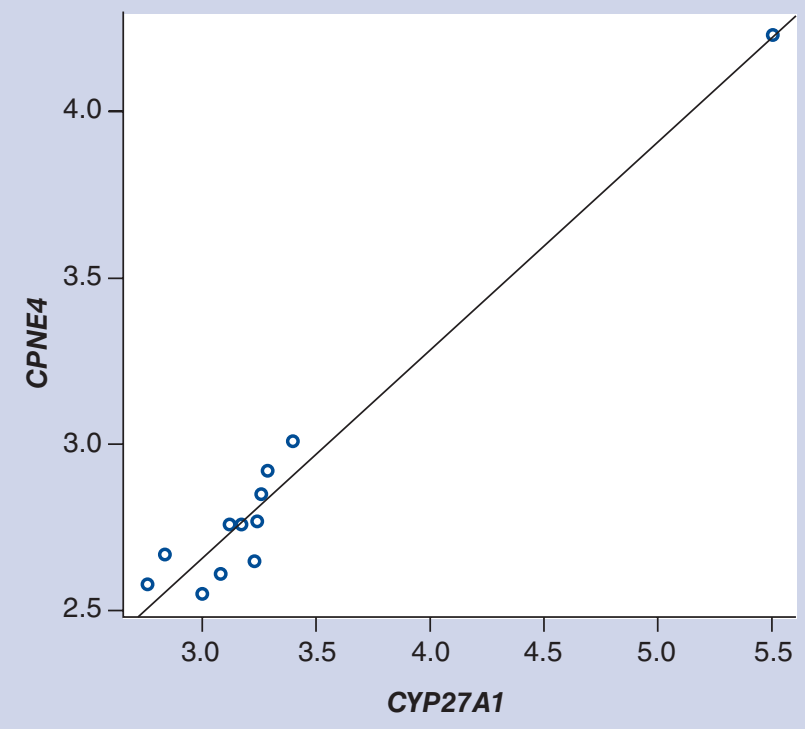

Figure 2. Pearson correlation plots for the expression levels of four pairs of genes in human lymphoblastoid cell lines from 12 unrelated Caucasian female donors. Gene names are followed by their chromosomal locations; note that pair members in these examples are located on different chromosomes (A) CHL1 (3p26.1) and CRIM1 (2p21). (B) CREB5 (7p15.1) and IFIH1 (2q24).

(C) SLC39A10 (2q32.3) and MYO1F (19p13.3). (D) CPNE4 (3q22.1) and CYP27A1 (2q33-qter)

code for ribosomal proteins; by comparison, the expression levels for GUSB, which has been proposed as the most appropriate reference gene for expression studies in LCLs [38], show in our data set slightly higher variations with mean $\pm \mathrm{SD}$ values of $7.73 \pm 0.16$ (Supplementary Table 2 ). We therefore propose that the ribosomal protein genes RPL5, RPL10 and RPL18 should also be considered as reference for expression studies in LCLs.
Microarray gene-expression data have been used for constructing gene interaction networks in Drosophila melanogaster [39] and Saccharomyces cerevisiae [40]. These studies employed time-course or single cell data from microarray experiments. However, to our knowledge, genome-wide microarray expression data sets from cell lines representing unrelated individuals have not been employed for exploring pairwise gene-expression correlations and the possible involvement of miRNAs in 


\section{Table 3. Pairs of genes with correlated expression levels and shared miRNA binding sites.}

\begin{tabular}{|c|c|c|c|c|c|}
\hline \multirow[t]{2}{*}{ First gene } & \multirow{2}{*}{$\begin{array}{l}\text { Second } \\
\text { gene }\end{array}$} & \multirow[t]{2}{*}{$r^{2}$} & \multicolumn{3}{|c|}{ Shared miRNAs } \\
\hline & & & DIANA & TargetScan & DIANA and TargetScan \\
\hline HNF1B & EPS8 & 0.9829 & $\begin{array}{l}\text { miR-92b, miR-92a, } \\
\text { miR-200c, miR-200b, } \\
\text { miR-32 }\end{array}$ & $\begin{array}{l}\text { miR-200b, miR-200c, miR-429, } \\
\text { miR-25, miR-32, miR-92a, miR-92b, } \\
\text { miR-363, miR-367 }\end{array}$ & $\begin{array}{l}\text { miR-92a, miR-92b } \\
\text { miR-200b, miR-200c, miR-32 }\end{array}$ \\
\hline NCKAP5 & CREB5 & 0.9768 & & $\begin{array}{l}\text { miR-29a, miR-29b, miR-29c, } \\
\text { miR-130a, miR-130b, miR-301a, } \\
\text { miR-301b, miR-454, miR-4295, } \\
\text { miR-3666, miR-19a, miR-19b, } \\
\text { miR-17, miR-20a, miR-20b, miR-93, } \\
\text { miR-106a, iR-106b, miR-519d }\end{array}$ & \\
\hline LMO3 & $L R R K 2$ & 0.9759 & $\begin{array}{l}\text { miR-181c, miR-181a, } \\
\text { miR-181b, miR-181d }\end{array}$ & $\begin{array}{l}\text { miR-181a, miR-181b, miR-181c, } \\
\text { miR-181d, miR-4262 }\end{array}$ & $\begin{array}{l}\text { miR-181a, miR-181b, } \\
\text { miR-181c, miR-181d }\end{array}$ \\
\hline CRIM1 & $\mathrm{CHL1}$ & 0.9755 & miR-369-3p, miR-570 & $\begin{array}{l}\text { miR-28-5p, miR-708, miR-3139, } \\
\text { miR-570 }\end{array}$ & miR-570 \\
\hline PXDN & RPS6KA6 & 0.9681 & & miR-9, miR-488 & \\
\hline BEND4 & ATP1B1 & 0.9673 & $\begin{array}{l}\text { miR-9, miR-101, miR-181a, } \\
\text { miR-181b, miR-181c, } \\
\text { miR-181d, miR-522 }\end{array}$ & $\begin{array}{l}\text { miR-221, miR-222, miR-9, miR-543, } \\
\text { miR-141, miR-200a, miR-101 }\end{array}$ & miR-9, miR-101 \\
\hline NOTCH2 & $C A M K 2 D$ & 0.9662 & $\begin{array}{l}\text { miR-30a, miR-30b, miR- } \\
\text { 30c, miR-30d, miR-30e, } \\
\text { miR-186, miR-211, miR-204 }\end{array}$ & $\begin{array}{l}\text { miR-214, miR-761, miR-3619-5p, } \\
\text { miR-204, miR-211, miR-539 }\end{array}$ & miR-204, miR-211 \\
\hline DLGAP1 & BTBD3 & 0.9659 & $\begin{array}{l}\text { miR-181a, miR-181b, } \\
\text { miR-181c, miR-181d, let-7e, } \\
\text { let-7a, let-7b, let-7c, let-7g, } \\
\text { let-7i, let-7f, miR-148b, } \\
\text { miR-148a, miR-152, } \\
\text { miR-98, miR-524-5p, } \\
\text { miR-520d-5p, miR-543 }\end{array}$ & $\begin{array}{l}\text { let-7a, let-7e, let-7b, let-7c, let-7d, } \\
\text { let-7g, let-7i, let-7f, miR-98, } \\
\text { miR-4458, miR-4500, miR-148a, } \\
\text { miR-148b, miR-152 }\end{array}$ & $\begin{array}{l}\text { let-7a, let-7e, let-7b, let-7c, } \\
\text { let-7g, let-7i, let-7f, miR-98, } \\
\text { miR-148a, miR-148b, miR-152 }\end{array}$ \\
\hline TNFRSF21 & PLD1 & 0.9651 & $\begin{array}{l}\text { miR-519b-3p, miR-519a, } \\
\text { miR-519c-3p, miR-9 }\end{array}$ & & \\
\hline HOXB2 & $D D \times 60$ & 0.9626 & miR-340 & & \\
\hline HOOK1 & PLD1 & 0.9615 & miR-203 & & \\
\hline IFIT5 & FGL2 & 0.9601 & miR-590-3p, miR-340 & & \\
\hline IL7 & EPS8 & 0.9587 & miR-137, miR-506 & & \\
\hline MED13L & LMO3 & 0.9584 & $\begin{array}{l}\operatorname{miR}-300, \operatorname{miR}-381 \\
\text { miR-495 }\end{array}$ & $\begin{array}{l}\text { miR-302a, miR-302b, miR-302c, } \\
\text { miR-302d, miR-302e, miR-372, miR- } \\
\text { 373, miR-520b, miR-520e, miR- } \\
\text { 520a-3p, miR-520c-3p, miR-520d- } \\
\text { 3p, miR-590-3p, miR-300, miR-381 }\end{array}$ & miR-300, miR-381 \\
\hline SLC39A10 & IPCEF1 & 0.9575 & $\begin{array}{l}\text { miR-23a, miR-23b, } \\
\text { miR-495, miR-543 }\end{array}$ & miR-23a, miR-23b, miR-23c & miR-23a, miR-23b \\
\hline CPEB3 & HNRPLL & 0.9568 & miR-340 & & \\
\hline CLEC4D & TEAD1 & 0.9551 & & miR-590-3p & \\
\hline IFIT5 & BEND4 & 0.9538 & miR-590-3p, miR-374a & miR-374a, miR-374b & miR-374a \\
\hline$X Y L T 1$ & ACTN1 & 0.9528 & miR-19b, miR-19a & miR-19a, miR-19b & miR-19a, miR-19b \\
\hline MBOAT2 & CREB5 & 0.9527 & $\begin{array}{l}\text { miR-579, miR-181b, } \\
\text { miR-181d, miR-181a, } \\
\text { miR-181c, miR-137, } \\
\text { miR-338-5p, miR-200b, } \\
\text { miR-200c }\end{array}$ & $\begin{array}{l}\text { miR-200b, miR-200c, miR-429, } \\
\text { miR-143, miR-4770, miR-137, } \\
\text { miR-33a, miR-33b }\end{array}$ & $\begin{array}{l}\text { miR-137, miR-200b, } \\
\text { miR-200c }\end{array}$ \\
\hline
\end{tabular}


Table 4. Genes with high expression

levels and extremely low variation among individual human lymphoblastoid cell lines.

\begin{tabular}{|c|c|c|}
\hline Gene & Mean $^{\dagger}$ & SD \\
\hline GDPH & 12.68 & 0.060 \\
\hline EEF1A1 & 12.54 & 0.038 \\
\hline ENO1 & 12.34 & 0.078 \\
\hline RPS4X & 12.26 & 0.061 \\
\hline RPL5 & 12.21 & 0.067 \\
\hline PTMA & 12.18 & 0.095 \\
\hline RPL10 & 12.18 & 0.080 \\
\hline$H L A-A$ & 12.05 & 0.072 \\
\hline RN7SK & 12.00 & 0.068 \\
\hline RPL18 & 12.00 & 0.067 \\
\hline \multicolumn{3}{|c|}{$\begin{array}{l}\text { Means and SD expression values are presented for genes } \\
\text { with highest expression levels and smallest variations } \\
\text { among the } 12 \text { examined individual human lymphoblastoid } \\
\text { cell lines. Such genes may be useful as reference genes for } \\
\text { real-time PCR studies. } \\
{ }^{+} \text {Expression values are shown in arbitrary Affymetrix units } \\
\text { in log }{ }_{2} \text { scale (see text). For further genes with high } \\
\text { expression levels see SupplemENTARY TABLE } 2 \text {. } \\
\text { SD: Standard deviation. }\end{array}$} \\
\hline
\end{tabular}

such regulatory events. Further research is warranted on the potential use of LCLs microarray transcript data sets for studying gene networks and their intricate regulation by miRNAs.

\section{Acknowledgements}

The authors thank the anonymous donors of the National Laboratory for the Genetics of Israeli Populations biobank at Tel Aviv University, Israel, whose altruism and trust in biomedical research have made this study possible. We thank Ofer Isakov for helpful computational analysis and Alice Chort for helpful suggestions.

\section{Financial \& competing interests disclosure}

This study was supported by the Chief Scientist Office, Ministry of Health, Israel, in the frame of ERA-Net Neuron and by the Shalom and Varda Yoran Institute for Human Genome Research at Tel Aviv University. The work of $M$ Vincent was supported by the BioIntelligence research project. N Shomron is supported by the NIH (R01DC011835); Chief Scientist Office, Ministry of Health, Israel; Israel Cancer Association; Wolfson Family Charitable Fund; I-CORE Program of the Planning and Budgeting Committee, The Israel Science Foundation, Israel (grant number 41/11); Claire and Amedee Maratier Institute for the Study of Blindness and Visual Disorders, Sackler Faculty of Medicine, Tel-Aviv University. The authors have no other relevant affiliations or financial involvement with any organization or entity with a financial interest in or financial conflict with the subject matter or materials discussed in the manuscript apart from those disclosed.

No writing assistance was utilized in the production of this manuscript

\section{Executive summary}

\section{Background}

- Human lymphoblastoid cell lines (LCLs) are a rich resource for information on human interindividual variations, including transcriptomic variations, and are therefore commonly used in pharmacogenomic studies. In this article we demonstrate that genome-wide transcriptome data of LCLs from a relatively small set of unrelated healthy individuals may detect pairs of genes that exhibit highly correlated expression levels and may thus convey insights about coregulated genes and thereby about cellular pathways.

\section{Materials \& methods}

- RNA samples were prepared from LCLs representing 12 unrelated healthy adult female Caucasian donors and grown in parallel under optimal conditions. Transcript levels were determined with the Affymetrix GeneChip ${ }^{\circledR}$ Human Gene 1.0 ST arrays. Data were normalized and analyzed for expression levels correlations, and the variations of each of approximately 33,000 transcripts in these 12 LCLs were calculated.

\section{Results \& discussion}

- When comparing the expression levels of each of the top 1000 genes exhibiting the largest interindividual expression-level variations against each of the other top 999 genes, two sets of highly correlated pairs with false-discovery rates controlled at $p<0.01$ and $p<0.05$ were detected, containing 157 and 4438 gene pairs, respectively. Performing the same analysis for another gene-expression data set obtained from human LCLs (GSE11582) detected 61 and 39\% of the same pairs as identified from our LCLs gene-expression data $(p<0.01$ and $p<0.05$, respectively), indicating high reproducibility between this and the previous data sets.

- Sequences of detected gene pairs were compared for the presence of conserved 3'-UTR microRNA binding sites which may in part explain their correlated expression levels. Shared conserved 3'-UTR miRNA binding sites were noted for 14-17\% of the top 100 pairs of strongly correlated genes in our data sets, suggesting that coregulation by miRNAs may contribute to their coordinated expression levels.

" Our data suggest that that the ribosomal protein genes RPL5, RPL10 and RPL18 should be considered as reference for expression studies in human LCLS.

- Transcriptomic data sets from LCLs representing unrelated healthy individuals may be useful for identifying coregulated genes, as well as clues for their coregulation by shared miRNAs. Such data sets may therefore add insights on cellular pathway regulation by miRNAs and on the implications of interindividual transcriptomic variations in pharmacogenomic differences between individuals. 


\section{Ethical conduct of research}

The authors state that they have obtained appropriate institutional review board approval or have followed the principles outlined in the Declaration of Helsinki for all human or animal experimental investigations. In addition, for investigations involving human subjects, informed consent has been obtained from the participants involved.

\section{References}

1 Cann HM, de Toma C, Cazes L et al. A human genome diversity cell line panel. Science 296(5566), 261-262 (2002).

2 Sie L, Loong S, Tan EK. Utility of lymphoblastoid cell lines. J. Neurosci. Res. 87(9), 1953-1959 (2009).

3 Wheeler HE, Dolan ME. Lymphoblastoid cell lines in pharmacogenomic discovery and clinical translation. Pharmacogenomics 13(1), 55-70 (2012).

4 Nam HY, Shim SM, Han BG, Jeon JP. Human lymphoblastoid cell lines: a goldmine for the biobankomics era. Pharmacogenomics 12(6), 907-917 (2011).

5 Stark AL, Zhang W, Mi S et al. Heritable and non-genetic factors as variables of pharmacologic phenotypes in lymphoblastoid cell lines. Pharmacogenomics J. 10(6), 505-512 (2010).

6 Woo JH, Cho SB, Lee E, Kim JH. Identifying regulatory relationships among genomic loci, biological pathways, and disease. Artif. Intell. Med. 49(3), 161-165 (2010).

7 Cheung VG, Conlin LK, Weber TM et al. Natural variation in human gene expression assessed in lymphoblastoid cells. Nat. Genet. 33(3), 422-425 (2003).

8 Coulombe-Huntington J, Lam KC, Dias C, Majewski J. Fine-scale variation and genetic determinants of alternative splicing across individuals. PLoS Genet. 5(12), e1000766 (2009).

9 Bleibel WK, Duan S, Huang RS et al. Identification of genomic regions contributing to etoposide-induced cytotoxicity. Hum. Genet. 125(2), 173-180 (2009).

10 Dolan ME, Newbold KG, Nagasubramanian $\mathrm{R}$ et al. Heritability and linkage analysis of sensitivity to cisplatin-induced cytotoxicity. Cancer Res. 64(12), 4353-4356 (2004).

11 Hartford CM, Duan S, Delaney SM et al. Population-specific genetic variants important in susceptibility to cytarabine arabinoside cytotoxicity. Blood 113(10), 2145-2153 (2009).

12 Huang RS, Duan S, Bleibel WK et al. A genome-wide approach to identify genetic variants that contribute to etoposide-induced cytotoxicity. Proc. Natl Acad. Sci. USA 104(23), 9758-9763 (2007).
13 Pinto N, Ludeman SM, Dolan ME. Drug focus: pharmacogenetic studies related to cyclophosphamide-based therapy. Pharmacogenomics 10(12), 1897-1903 (2009).

14 Li L, Fridley B, Kalari K et al. Gemcitabine and cytosine arabinoside cytotoxicity: association with lymphoblastoid cell expression. Cancer Res. 68(17), 7050-7058 (2008).

15 Niu N, Qin Y, Fridley BL et al. Radiation pharmacogenomics: a genome-wide association approach to identify radiation response biomarkers using human lymphoblastoid cell lines. Genome Res. 20(11):1482-1492 (2010).

16 Choy E, Yelensky R, Bonakdar S et al. Genetic analysis of human traits in vitro: drug response and gene expression in lymphoblastoid cell lines. PLoS Genet. 4(11), e1000287 (2008).

17 Morag A, Kirchheiner J, Rehavi M, Gurwitz D. Human lymphoblastoid cell line panels: novel tools for assessing shared drug pathways. Pharmacogenomics 11(3), 327-340 (2010).

18 Morag A, Pasmanik-Chor M, Oron-Karni V et al. Genome-wide expression profiling of human lymphoblastoid cell lines identifies CHL1 as a putative SSRI antidepressant response biomarker. Pharmacogenomics 12(2), 171-184 (2011).

19 Oved K, Morag A, Pasmanik-Chor M et al. Genome-wide miRNA expression proiling of human lymphoblastoid cell lines identifies tentative SSRI antidepressants response biomarkers. Pharmacogenomics 13(10), 1129-1139 (2012).

20 Morag A, Oved K, Gurwitz D. Sex Differences in Human Lymphoblastoid Cells Sensitivities to Antipsychotic Drugs. J. Mol. Neurosci. doi:10.1007/s12031-012-9852-z (2012) (Epub ahead of print).

21 Nishimura Y, Martin CL, Vazquez-Lopez A, et al. Genome-wide expression profiling of lymphoblastoid cell lines distinguishes different forms of autism and reveals shared pathways. Hum. Mol. Genet. 16(14), 1682-1698 (2007).

22 Eisen MB, Spellman PT, Brown PO, Botstein D. Cluster analysis and display of genomewide expression patterns. Proc. Natl Acad. Sci. USA 95(25), 14863-14868 (1998).

23 Opgen-Rhein R, Strimmer K. Learning causal networks from systems biology time course data: an effective model selection procedure for the vector autoregressive process. BMC Bioinformatics 8(Suppl. 2) S3 (2007).

24 Zhu D, Hero AO, Qin ZS, Swaroop A. High throughput screening of co-expressed gene pairs with controlled false discovery rate
(FDR) and minimum acceptable strength (MAS). J. Comput. Biol. 12(7), 1029-1045 (2005).

25 Chomczynski P. A reagent for the single-step simultaneous isolation of RNA, DNA and proteins from cell and tissue samples. Biotechniques 15(3), 532-534, 536-537 (1993).

26 Benjamini Y, Hochberg Y. Controlling the false discovery rate: a practical and powerful approach to multiple testing. J. Roy. Statist. Soc. B 57, 289-300 (1995).

27 Maragkakis M, Alexiou P, Papadopoulos GL et al. Accurate microRNA target prediction correlates with protein repression levels. $B M C$ Bioinformatics 10, 295 (2009).

28 Maragkakis M, Reczko M, Simossis VA et al. DIANA-microT web server: elucidating microRNA functions through target prediction. Nucleic Acids Res. 37(Web Server issue), W273-276 (2009).

29 Razick S, Magklaras G, Donaldson IM. iRefIndex: a consolidated protein interaction database with provenance. BMC Bioinformatics 9, 405 (2008).

30 Jensen LJ, Kuhn M, Stark M et al. STRING 8 --a global view on proteins and their functional interactions in 630 organisms. Nucleic Acids Res. 37(Database issue), D412-416 (2009).

31 Szklarczyk D, Franceschini A, Kuhn M et al. The STRING database in 2011: functional interaction networks of proteins, globally integrated and scored. Nucleic Acids Res. 39(Database issue), D561-8 (2011).

32 Berezikov E. Evolution of microRNA diversity and regulation in animals. Nat. Rev. Genet. 12(12), 846-860 (2011).

33 Esteller M. Non-coding RNAs in human disease. Nat. Rev. Genet. 12(12), 861-874 (2011).

34 Rukov JL, Shomron N. MicroRNA pharmacogenomics: post-transcriptional regulation of drug response. Trends Mol. Med. 17(8), 412-423 (2011).

35 Huang X, Zhu LL, Zhao T et al. CHL1 negatively regulates the proliferation and neuronal differentiation of neural progenitor cells through activation of the ERK1/2 MAPK pathway. Mol. Cell. Neurosci. 46(1), 296-307 (2011).

36 Wilkinson L, Kolle G, Wen D, Piper M, Scott J, Little M. CRIM1 regulates the rate of processing and delivery of bone morphogenetic proteins to the cell surface. J. Biol. Chem. 278(36), 34181-34188 (2003).

37 Zhang W, Huang RS, Duan S, Dolan ME. Gene set enrichment analyses revealed differences in gene expression patterns between males and females. In Silico Biol. 9(3), 55-63 (2009). 
de Brouwer AP, van Bokhoven $\mathrm{H}$, Kremer $\mathrm{H}$

Comparison of 12 reference genes for normalization of gene expression levels in Epstein-Barr virus-transformed

lymphoblastoid cell lines and fibroblasts. Mol. Diagn. Ther. 10(3), 197-204 (2006).

39 Gowda T, Vrudhula S, Kim S. Prediction of pairwise gene interaction using threshold logic. Ann. N Y Acad. Sci. 1158, 276-286 (2009).

40 Wyart M, Botstein D, Wingreen NS. Evaluating gene expression dynamics using pairwise RNA FISH data. PLoS Comput. Biol. 6(11), e1000979 (2010).

\section{Websites}

101 National Laboratory for the Genetics of Israeli Populations (NLGIP) at Tel-Aviv University. http://nlgip.tau.ac.il

102 Affymetrix GeneChip ${ }^{\circledR}$ Human Gene 1.0 ST arrays.

www.fleming.gr/expu/gene_1_0_st_ datasheet.pdf

103 Partek $^{\circledR}$ Genomics Suite ${ }^{\mathrm{TM}}$. www.partek.com/

104 Human Genome Organisation (HUGO) gene nomenclature.

www.genenames.org/
105 DIANA LAB: DNA Intelligent Analysis, DIANA microT v3.0 software. www.microrna.gr/microT

106 TargetScanHuman 5.2. www.targetscan.org/vert_50/

107 Gene Expression Omnibus. www.ncbi.nlm.nih.gov/geo/

108 NCBI Gene Expression Omnibus dataset GSE11582.

www.ncbi.nlm.nih.gov/geo/query/acc. cgi?acc $=$ GSE 11582

109 iRefIndex (index of protein interactions). http://irefindex.uio.no/wiki/iRefIndex

110 Ig. STRING - Known and Predicted Protein-Protein Interactions. http://string-db.org/ 